\title{
Manipulation of ultracold atomic mixtures using microwave techniques
}

\author{
D. Ciampini, E. Courtade, C. Sias, D. Cossart, G. Carelli, \\ F. Mango, O. Morsch, E. Arimondo \\ INFM, Dipartimento di Fisica E.Fermi, Università di Pisa, Largo Pontecorvo 3, \\ I-56127 Pisa,Italy
}

\begin{abstract}
We used microwave radiation to evaporatively cool a mixture of of ${ }^{133} \mathrm{Cs}$ and ${ }^{87} \mathrm{Rb}$ atoms in a magnetic trap. A mixture composed of an equal number (around 10 ${ }^{4}$ ) of $\mathrm{Rb}$ and $\mathrm{Cs}$ atoms in their doubly polarized states at ultracold temperatures was prepared. We also used microwaves to selectively evaporate atoms in different Zeeman states.
\end{abstract}

Key words: alkali mixture microwave evaporation microwave selection PACS: $32.80 . \mathrm{Pj}, 32.10 . \mathrm{Fn}$, 84.40.Ba

\section{Introduction}

Laser cooling techniques, combined with evaporative cooling in magnetic traps, allow the Bose-Einstein condensation (BEC) of a dilute atomic gas [1]. Typically, the atoms are trapped in conservative magnetic potentials and ultracold temperatures are reached through evaporative cooling using, for instance, radiofrequency (rf), microwave (mw) or circle-of death techniques. The evaporation methods have different applicability and efficiency, which is more evident when dealing with combinations of ultra-cold atoms. A few combinations of ultra-cold atoms have been studied in conservative traps, among them LiCs [2], K-Rb [3,4,5], and Na-Li [6,7].

In experiments on ultracold atoms and atomic mixtures the use of mw radiation in evaporative cooling has been explored by a number of groups and has become a commonly used technique in the field. For example, controlled state selective evaporation of a single species was applied in [8]. Selective evaporative cooling of a single species in a two-species magnetic trap was used in Ref. $[4,9,10]$, for the sympathetic cooling of ${ }^{6} \mathrm{Li}$ by ${ }^{7} \mathrm{Li}$ in $[11]$ and for sympathetic 
cooling of ${ }^{6} \mathrm{Li}$ by ${ }^{23} \mathrm{Na}$ in [6]. We have applied mw evaporation to a mixture of ultra-cold ${ }^{87} \mathrm{Rb}$ and ${ }^{133} \mathrm{Cs}$ atoms in a magnetic trap in order to explore sympathetic cooling and the collisional rethermalization between the two species, as reported in Ref. [12]. With the aim of producing a very cold Cs sample, we explored the temperature limits associated with circle-of-death, rf and mw evaporation. This detailed exploration is the topic of the present work.

Our best approach for evaporating the Rb-Cs mixture was the sequential application of different evaporative cooling processes: an initial stage of circle-ofdeath evaporation active on both species, followed by direct mw evaporative cooling of $\mathrm{Cs}$, and finally rf cooling of Rb that led to sympathetic cooling of Cs through collisional energy exchange with Rb atoms. Applying the usual sequence of circle-of-death followed by radio-frequency evaporation for achieving BEC in a TOP trap, we verified that, to a good approximation, circle-of-death evaporation is equally efficient on $\mathrm{Cs}$ and $\mathrm{Rb}$ atoms. We discovered that owing to the large Cs-Rb interspecies scattering length $a_{R b-C s} \simeq 590 a_{0}[12]$ sympathetic cooling of Cs atoms by collisions with $\mathrm{Rb}$ is a very effective process. However, the Rb atom population is substantially depleted before very low Cs temperatures are achieved. Therefore, in order to reach very low Cs temperatures, we introduced an intermediate stage of mw evaporation. This sequence of different evaporation techniques was optimised in order to allow us to measure the $\mathrm{Cs}-\mathrm{Rb}$ relative scattering length at a temperature of $6 \mu \mathrm{K}$, as we reported in [12]. In the case where rf evaporation of $\mathrm{Rb}$ was applied without the intermediate step of microwave evaporation, the lowest temperature achieved for performing the scattering length measurement was at least two times larger.

While characterizing the Rb-Cs ultracold mixture we faced the presence of ultracold atoms in Zeeman sublevels different from the desired doubly polarized state. For several degenerate gas investigations, an incomplete atomic magnetization represents a great difficulty. Therefore, we employed a mw irradiation technique to selectively evaporate $\mathrm{Rb}$ or Cs atoms in specific Zeeman sublevels[13]. While rf evaporation drives transitions between all the Zeeman sublevels of a hyperfine state, the applied mw evaporation acts only on a given Zeeman sublevel. We report magnetization measurements for the $\mathrm{Rb}$ and $\mathrm{Cs}$ atoms before and after selective mw evaporation. This magnetization purification process could be useful for exploring the dependence of the scattering length on the Zeeman state.

This manuscript is organized as follows: Section II describes the experimental setup used for the preparation of the cold Rb-Cs mixture, the generation of mw radiation and its delivery to the atomic sample and mw spectroscopy within the magnetic trap. The evaporative cooling of the atomic mixture by mw radiation is described in Section III, while the state-selective removal of atoms is in Section IV. Section V presents some conclusions. 


\section{Experimental setup and techniques}

\subsection{Optical components}

We used a double-chamber vacuum system with a 2D collection MOT and a six-beam MOT [15,12]. Once the two species MOT was filled, after brief compressed MOT and molasses phases the trapping beams were switched off and the atoms were optically pumped into the $\left|F=2, m_{F}=2\right\rangle$ and $\left|F=4, m_{F}=4\right\rangle$ doubly polarized states of $\mathrm{Rb}$ and $\mathrm{Cs}$, respectively. Immediately after that, the TOP magnetic trap was switched on. The bias field $B_{0}$ of the TOP rotating at $10 \mathrm{KHz}$ in the horizontal plane was created by two pairs of coils, fed by a common function generator through a 90 degree phase shifter. A schematic of the coils for the TOP trap is presented in Fig. 1.

The temperature of the atomic mixture was then lowered first by circle-of-

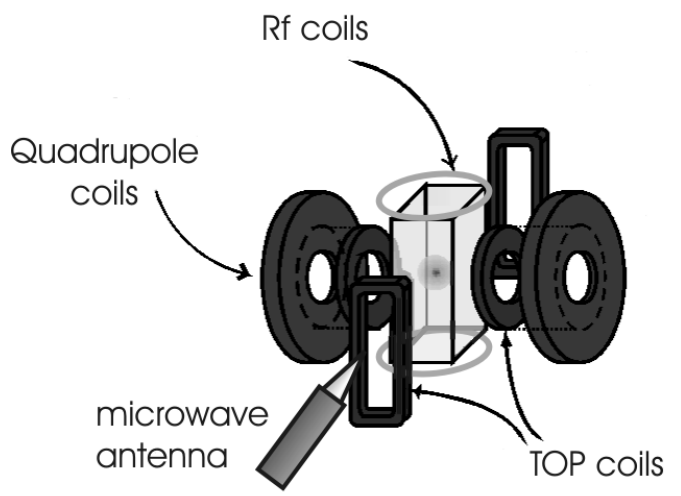

Fig. 1. Schematic of the coils for the magnetic trap. The quadrupole axis is along the horizontal direction while the bias field, produced by the TOP coils, rotates in the horizontal plane. The rf coils and the mw antenna are visible.

death evaporation and afterwards by radiative evaporation (rf and/or mw radiation). The atom number and temperature were measured by flashing on a beam resonant with one of the atomic species and recording the resulting shadow cast on a CCD camera by the atom cloud. For a given atom, different Zeeman sublevels can be magnetically trapped and, after switching off the magnetic trap, all of them were imaged by the same resonant laser flash. In order to observe their relative populations for $\mathrm{Rb}$ we used a very shallow magnetic potential in order to maximize the spatial separation along the vertical direction between the equilibrium positions of the sublevels [15], the so called gravity sag. For Cs atoms it was not possible to spatially distinguish the atoms in the $\left|F=4, m_{F}=4\right\rangle$ Zeeman sublevel from those in the $\left|F=4, m_{F}=3\right\rangle$ state using the differential gravity sag, the ratio of the magnetic moments of the two trapped states being only $\frac{4}{3}$, compared to the value of 2 for Rb. Thus in order to monitor the Cs atoms in different sublevels as separate clouds, we 
performed a Stern-Gerlach type experiment (3ms in which the atoms are in the quadrupole field only) separating the Zeeman levels in time-of-flight.

\subsection{Microwave source}

The mw radiation was produced by a frequency locked oscillator system composed of a Sweep Oscillator (HP 8350B), a Frequency Counter (HP 5343A) and a Source Synchronizer (HP 5344A). The frequency sweep and the mw switch were computer-controlled and synchronized to the experimental cycle. The mw radiation was amplified up to $5 \mathrm{~W}$ by a second power amplifier (Kuhne, model KU702 for the C-band, 4-8 GHz, and model KU922 for the Xband, 8-12 GHz). The mw radiation was delivered to the atoms by a dielectric rod antenna. In our experiment we used two different antennas, one made of teflon, optimized to deliver radiation at $9.2 \mathrm{GHz}$ and one made of plexiglass, used in the region around $6.8 \mathrm{GHz}$. The antennas consist of a circular-section dielectric rod directly inserted into the circular end of a metal waveguide for the $\mathrm{X}(\mathrm{Cs})$ and $\mathrm{C}(\mathrm{Rb})$ bands. The rod is conically shaped at both ends, with the cone lengths equal to $6 \mathrm{~cm}$ and $8 \mathrm{~cm}$ for the $\mathrm{Cs}$ and $\mathrm{Rb}$ antennas, respectively. Such linearly tapered-rod antennas have been extensively studied, and the presence of a dielectric close to the conductor structure profoundly modifies the performance of the antenna [16].

In a preliminary experiment we verified the behavior of the mw antennas by monitoring the reflection coefficient with a network analyzer over a band of frequencies around the desired frequency. For both antennas, the reflected

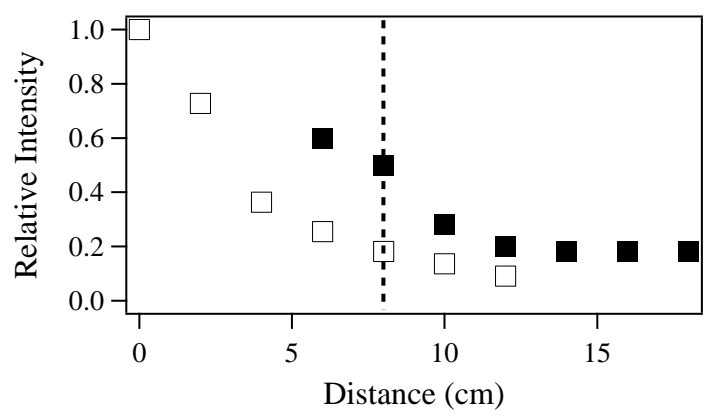

Fig. 2. Relative intensity of the e.m. field measured at various distances from the end of the X-band metal waveguide, in the presence (solid squares) and in the absence (open squares) of the dielectric rod extension. The dashed line marks the distance where the cold atoms are located during the experiment.

power fraction never exceeded $-10 \mathrm{~dB}$, with a modulation structure related to the presence of objects adjacent to the antenna. The effect of the dielectric rod is to concentrate the mw field in a lobe in the direction of the cone termination [17], and the directivity gain is determined primarily by the antenna 
length. We experimentally found that for the same on-axis distance from the conductor waveguide, the field intensity was amplified by a factor 2.4 when the dielectric rod was inserted (see fig. 2). We also noticed a large reflection of the mw radiation due to metallic objects located in front of the antenna.

\subsection{Microwave spectroscopy}

We performed mw precision spectroscopy of the trapped $\mathrm{Rb}$ and $\mathrm{Cs}$ atoms by inducing transitions between their hyperfine levels $\left|F=I+1 / 2, m_{F}=I+1 / 2\right\rangle$ and $\left|F=I-1 / 2, m_{F}=I-1 / 2\right\rangle$. As pointed out previously $[18,19,20]$, for a cloud of magnetically trapped atoms the inhomogeneity due to the energy level shifts broadens the transition frequency and limits the attainable precision. Furthermore, in a TOP trap the presence of the time-varying field of the trap introduces a time-varying detuning that greatly complicates the interaction between trapped atoms and mw radiation [21]. Our mw spectroscopical investigation within the TOP trap for an interaction time long compared to the rotating field period demonstrated a strong dependence of the mw transition linewidth on the temporal variation of the modulus of the rotating bias field. The analysis of the condensate micromotion in the TOP [22] shows that the atoms follow an orbit corresponding to a constant total modulus of the magnetic field if the amplitude $B_{0}$ of the bias field is constant during the horizontal rotation. On the other hand, the total magnetic field experienced by the atoms during their motion contains components varying at the rotating field and its harmonics in presence of an elliptical rotating bias field. Such an elliptical bias field appears, for instance, if the two linearly oscillating magnetic fields whose superposition produces the rotating bias field are not very precisely matched in amplitude and phase. This behavior is confirmed by the data of Fig.3 for the number of Rb atoms remaining in the magnetic trap after $10 \mathrm{~s}$ application of $5 \mathrm{~W}$ mw radiation whose frequency was scanned over the resonant value. For a 30 percent ellipticity of the bias field we observed a well-defined double peak structure for the mw transition (open squares). When the ellipticity of the bias field was compensated by adjusting the relative value of the magnetic field amplitudes in the two pairs of TOP coils and the phase, the two peaks coincided (solid circles). This method is very sensitive: operating at our maximum amplitude of the bias field, a phase mismatch of 5 degrees between the two oscillating fields and an ellipticity of $10 \%$ broadens the mw transition by $4 \mathrm{MHz}$. We found this fine adjustment procedure of the bias field based on the linewidth of the mw transition to be more sensitive than the procedure based on the optimization of the circle-of-death evaporation efficiency. Moreover, we performed mw spectroscopy within the TOP trap for an interaction time short compared to the period of rotation of the TOP field, and finally performing spectroscopy in time of flight in the presence of a rotating bias field or a weak 


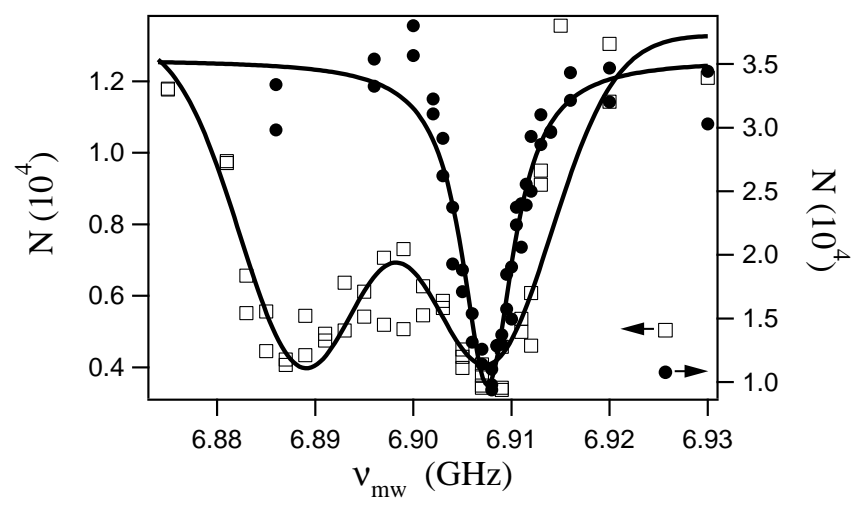

Fig. 3. Number of Rb atoms after application of mw radiation for $10 \mathrm{~s}$ in the TOP trap as a function of the mw frequency for two different choices of the relative amplitude of the oscillating fields of the TOP trap, leading to a 30 percent ellipticity (open squares, left axis) and to a circular rotating bias field, compensated in ellipticity and phase as described in the text (full circles, right axis). The full lines are double Gaussian and Lorentzian fits, respectively, to the data sets.

homogeneous magnetic field. In this sequence of experiments we eliminated the residual inhomoegenous broadening and measured a decreasing linewidth. The final limiting linewidth at full mw power, $5 \mathrm{~W}$ (the power injected into the waveguide), was around $100 \mathrm{kHz}$, in good agreement with the theoretical value obtained by estimating the amplitude of the mw magnetic field $(\sim 70$ $\mathrm{mG}$ ) at the position of the atoms from the power inserted into the waveguide and the enhancement factor due to the teflon cone (as seen in Fig. 2).

\section{Evaporation procedure}

\subsection{Circle of death}

In a TOP trap the temperature of the atomic mixture can be lowered through circle-of-death evaporative cooling, defined by the rotating zero of field created by the (static) quadrupole and the (rotating) bias field. By continuously reducing the strength of the rotating bias field the circle-of-death shrinks and atoms from the high energy tail of the distribution are progressively removed from the sample. In the double polarized Zeeman states Cs and Rb atoms have the same magnetic moment. Therefore, at the same temperature they have the same spatial extension in a magnetic trap, and circle-of-death evaporation is simultaneously applied to both species. At low temperatures and high atomic density, circle-of-death evaporation becomes inefficient because it requires a continuous increase of the trapping frequencies leading to an increased atomic density, resulting in large three-body losses. At the end of our circle-of-death 
evaporation phase, $\mathrm{Rb}$ and $\mathrm{Cs}$ atoms were in thermal equilibrium at $\sim 15 \mu \mathrm{K}$.

\subsection{Radiative evaporation: radiofrequency}

Radiative evaporation uses an e.m. radiation field to transfer atoms from a trapped to an untrapped state in an energy-selective way. The advantages of radiative evaporation ( $\mathrm{rf}$ and $\mathrm{mw}$ ) are that the magnetic potential does not have to be modified to sustain the evaporation since the escape rate is precisely controlled by the amplitude and the frequency of the applied radiation.

Rf induced evaporation between $\left|F, m_{F}=F\right\rangle$ and $\left|F, m_{F}=F-1\right\rangle$ states exploits spin-flips of atoms on a resonant energy shell defined by

$$
g_{F} \mu_{B} B(\vec{x})=\hbar \omega_{r f}
$$

where $B(\vec{x})$ is the modulus of the instantaneous local magnetic field defining the cut energy of the shell, $\mu_{B}$ is the Bohr magneton, $g_{F}=2 /(2 I+1)$ is the Landé factor, with $I=3 / 2$ for ${ }^{87} \mathrm{Rb}$ and $I=7 / 2$ for ${ }^{133} \mathrm{Cs}$, and $\omega_{r f}$ is the rf field frequency. Notice that Eq. 1, and the following one for the mw evaporation, is derived in the limit of weak Zeeman splitting. For $F>1$ the rf transition defined by Eq. (1) transfers atoms into a different Zeeman state that is still magnetically trapped. However, because of power broadening the rf radiation induces a chain of transitions between different Zeeman levels starting from trapped states to untrapped ones leading to a loss of atoms from the magnetic trap. By ramping down the frequency $\omega_{r f}$, the radius of the surface volume where the resonance condition is fulfilled shrinks, leading to an effective forced evaporation [1].

Eq. 1 specifies that rf evaporation depends on the Zeeman-sublevel spacing. When rf radiation is applied to a mixture of atoms, the cut energy of the atoms to be evaporated is different. In a Rb-Cs mixture, owing to the difference in the Landé factor, the Cs cut energy is half that for Rb atoms. Thus, when the most energetic $\mathrm{Cs}$ atoms are removed by the rf radiation, cold $\mathrm{Rb}$ atoms at the bottom of the potential are also removed. On the contrary, cooling the

$\mathrm{Rb}$ atoms with rf has no direct effect on the Cs temperature, because the $\mathrm{rf}$ field is not resonant with the trapped Cs atoms.

\subsection{Radiative mw evaporation}

For a mixture of atomic species mw evaporative cooling represents an efficient alternative to rf evaporative cooling. Mw evaporation uses transitions between Zeeman sublevels belonging to different hyperfine levels of the ground states. 


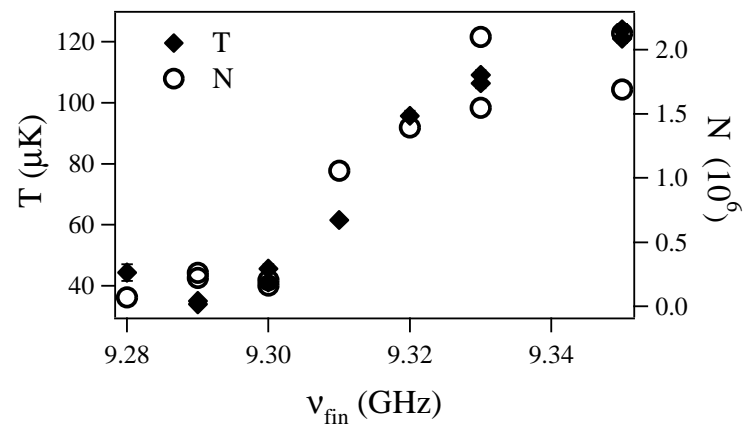

Fig. 4. Temperature (full diamonds) and number of atoms (open circles) of the Cs cloud as a function of the final value of the mw frequency. Initial frequency $9.40 \mathrm{GHz}$, trap bottom $9.271 \mathrm{GHz}$, duration of the evaporation ramp $5 \mathrm{~s}$ and mw power $3 \mathrm{~W}$. These data were from single experimental runs and the uncertainties due to the fit of the single images were smaller than the size of the data points.

For a transition between the hyperfine states $\left|F, m_{F}=F\right\rangle$ and $\mid F-1, m_{F}=$ $F-1\rangle$ of an alkali atom, the resonance condition is

$$
\hbar \omega_{h f}+g_{F}\left(2 m_{F}-1\right) \mu_{B} B(\vec{x})=\hbar \omega_{m w}
$$

where $\omega_{h f} / 2 \pi$ is the hyperfine splitting $(\sim 9.2 \mathrm{GHz}$ for $\mathrm{Cs}, \sim 6.8 \mathrm{GHz}$ for $\mathrm{Rb})$, and $\omega_{m w} / 2 \pi$ the mw frequency. The previous equation indicates that the $\mathrm{mw}$ resonance conditions for $\mathrm{Cs}$ and $\mathrm{Rb}$ are always very different and the two evaporation processes are independent.

Fig. 4 shows the temperature and atom number of the Cs cloud at the end of mw evaporation in the absence of $\mathrm{Rb}$ atoms in the magnetic trap. During the evaporative cooling the mean temperature of the Cs cloud decreased in direct proportion to the number of atoms. The cloud was irradiated by a mw frequency ramp with a fixed starting frequency and varying final frequency. The evaporation took place after a magnetic compression phase and a circleof-death cooling stage. The data points at the far right describe the initial condition before the application of the mw radiation. The dependence of the final temperature of the Cs cloud on the mw intensity is shown in Fig. 5. In our experiments we generally observed a lower efficiency for the mw evaporation compared to rf evaporation. Due to imperfect optical pumping (and, possibly, other depolarizing processes during the evaporation cycle), both the Rb and Cs cold clouds had admixtures of atoms in other Zeeman sublevels, as discussed in the following Section. While the radio-frequency radiation, inducing a chain of transitions between the equally spaced Zeeman sublevels, evaporates atoms from all of them, the mw transitions of Eq. 2 are well separated in frequency, at $30-40 \mathrm{G}$ trap field and in the absence of saturation broadening. Thus, the mw field is resonant with one Zeeman state only, leaving the residual population of the other sublevels uncooled by the mw radiation. This residual population is eventually cooled through sympathetic cooling collisions with the atoms in the 


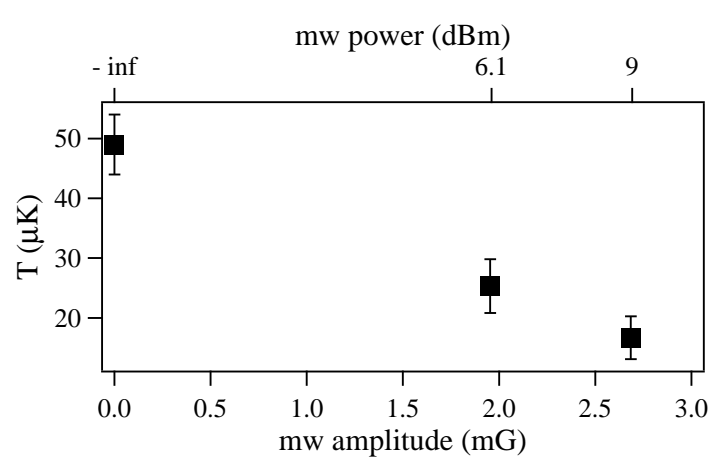

Fig. 5. Cs temperature as a function of the mw power (in the upper horizontal scale), and of the mw field amplitude at the atom position (in the lower scale), for a given evaporation ramp (from $9.3 \mathrm{GHz}$ to $9.22 \mathrm{GHz}$, trap bottom $9.215 \mathrm{GHz}$ ) with $5 \mathrm{~s}$ duration. The data uncertainty is due to statistical averaging over several experimental runs.

other Zeeman state, but the overall efficiency of the mw evaporation is reduced. From the data of Fig. 4 for Cs, assuming an atomic occupation of the single Zeeman sublevel $\left|F=4, m_{F}=4\right\rangle$, we derived that under mw evaporation the increase of the phase space density followed a $N^{-2}$ law with $N$ the atom number, a law similar to that required for the runaway regime reached by the rf evaporation [1]. However, the occupation of the $\left|F=4, m_{F}=3\right\rangle$ sublevel for the data in Fig. 4 is around 30-40\%, as extracted from the Stern-Gerlach type experiments performed with ultracold Cs atoms (see Section IV), and that occupation greatly decreases the effective efficiency of the phase-space compression by the mw evaporation.

\subsection{Sympathetic cooling}

After the preparative stages of circle-of-death and mw evaporation we verified that when performing rf evaporation on the $\mathrm{Rb}$ atoms, the measured temperature of the $\mathrm{Cs}$ atoms exactly followed the $\mathrm{Rb}$ temperature down to a few $\mu \mathrm{K}$, indicating that sympathetic cooling was taking place, as long as the remaining number of $\mathrm{Rb}$ atoms, compared to the Cs atom number, was sufficient to sustain the thermalization. By applying mw techniques we were able to prepare a mixture of $4 \times 10^{4} \mathrm{Rb}$ atoms in the $\left|F=2, m_{F}=2\right\rangle$ state and $10^{4} \mathrm{Cs}$ atoms in the $\left|F=4, m_{F}=4\right\rangle$ state at about $6 \mu \mathrm{K}$ (mean trapping frequency $70 \mathrm{~Hz}$ ). This temperature is of the same order of magnitude as that reported by the ENS group [24] for Cs in the same atomic state in their early search for condensation of Cs. From our data we extrapolated that for Cs a temperature around $3 \mu \mathrm{k}$ could be achieved if all of the Rb was evaporated. A detailed model of the sympathetic cooling phase was developed in ref. [12] in order to derive the Rb-Cs interspecies scattering length. 


\section{State selective evaporation}

Another application of the state selectivity provided by the mw evaporation technique is state purification. Since at the end of the circle-of-death evaporation phase we measured a percentage of around 80 percent of $\mathrm{Rb}$ atoms in the desired doubly polarized state $\left|F=2, m_{F}=2\right\rangle$, with around 20 percent in the $\left|F=2, m_{F}=1\right\rangle$ sublevel, we used the mw radiation to eliminate the populations in the sublevels other than the desired doubly polarized state. This is not achievable using rf radiation since, owing to saturation broadening, the resonance condition of Eq. 1 is equally fulfilled for atoms in all trapped Zeeman states. The results of the state selective evaporation in a $\mathrm{Rb}$ atomic sample is shown in Fig. 6. The two peaks in the upper record correspond to the absorption profile of $0.8 \mu \mathrm{K} \mathrm{Rb}$ atoms in the different Zeeman states, spatially separated along the vertical direction because of the differential sag. In (b) atoms in the $\left|F=2, m_{F}=1\right\rangle$ state have been reduced from 10 percent to an undetectable level, with no loss of atoms from the $\left|F=2, m_{F}=2\right\rangle$ state, when $100 \mathrm{~mW} \mathrm{mw}$ radiation at $6.858 \mathrm{GHz}$ resonant with the the hyperfine transition at the bottom of the magnetic trap was applied for $10 \mathrm{~s}$. Even if the mw radiation was not resonant with the whole sample because of inhomogeneous broadening, rethermalizing collisions allowed the evaporation to act on the whole hyperfine level occupation. In (c) mw radiation at $6.904 \mathrm{GHz}$ was applied to the $\left|F=2, m_{F}=2\right\rangle$ hyperfine level with a residual six percent final occupation in that state.

For Cs atoms, performing a Stern-Gerlach type experiment separating the Zeeman levels in time-of-flight at the end of the circle-of-death evaporation phase we detected relative populations of Cs atoms in the $\left|F=4, m_{F}=4\right\rangle$ Zeeman sublevel and in $\left|F=4, m_{F}=3\right\rangle$ of 60 percent and 40 percent, respectively. The percentage of atoms in the unwanted Zeeman sublevel was higher for Cs atoms than for Rb atoms. In Fig. 6 (d) the density profile of a Cs cloud is shown after the application of $5 \mathrm{~s}$ of mw radiation at $9.250 \mathrm{GHz}$ resonant with the $\left|F=4, m_{F}=3\right\rangle \rightarrow\left|F=3, m_{F}=2\right\rangle$ transition at the bottom of the trap, followed by a Stern-Gerlach phase, with a remaining fraction of $m_{F}=3$ atoms of $30 \%$. The number of Cs atoms before the application of the mw was around $10^{4}$ and their temperature $\sim 4 \mu \mathrm{K}$ at $51 \mathrm{~Hz}$ mean trapping frequency. By applying a $10 \mathrm{~s} \mathrm{mw}$ evaporation phase, similar to that applied to the $\mathrm{Rb}$ atoms, we were able to reduce the fraction of atoms in the $\left|F=4, m_{F}=3\right\rangle$ state to about 20 percent. The different Zeeman occupations for Rb and $\mathrm{Cs}$ that we found to start with in the magnetic trap and the different efficiency of the selective mw removal of atoms in the $\mathrm{Rb}$ and Cs case are compatible with the rate of density-dependent inelastic collisions of Cs atoms leading to a change of the $m_{F}$ quantum number, as reported by [23]. From our data taken at a Cs density of $8 . \times 10^{10} \mathrm{~cm}^{-3}$ we infer an inelastic rate coefficient around 1. $\times 10^{-12} \mathrm{~cm}^{3} \mathrm{~s}^{-1}$. This value is in agreement with the losses reported in the experiment of ref. [23] performed at a different magnetic field and a higher 


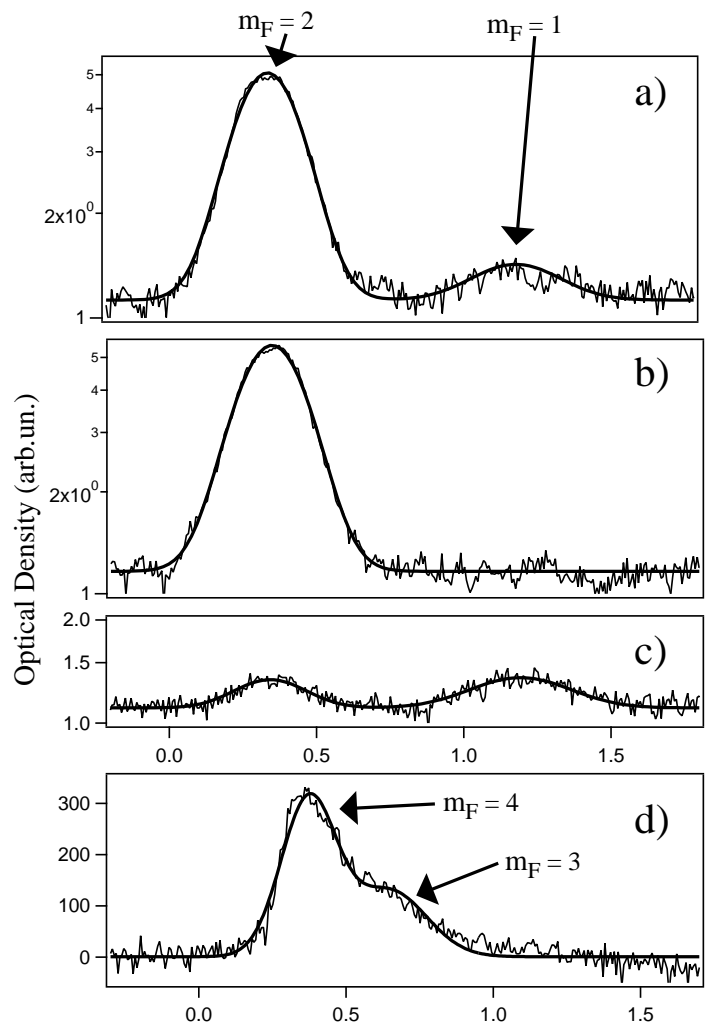

Position (mm)

Fig. 6. Rb integrated absorption profile in free fall (a) before and (b), (c) after selective mw evaporation, as in the text. The two peaks correspond to the $\left|F=2, m_{F}=2\right\rangle$ and $\left|F=2, m_{F}=1\right\rangle$ states experiencing a different gravitational sag for a mean trap frequency of $25.7 \mathrm{~Hz}$, with the atomic position measured downwards from the quadrupole symmetry center. In (b), (c) and (d) mw evaporative cooling produced a purification of $\mathrm{Rb}$ magnetization, as in the text. In (d), integrated absorption profile of Cs atoms, detected though a Stern-Gerlach type experiment, after selective removal of atoms in the $\left|F=4, m_{F}=3\right\rangle$ state, with the procedure explained in the text. Note that for better visibility we chose in (a), (b) and (c) a logarithmic vertical scale, while in (d) the vertical scale is linear.

temperature.

\section{Conclusions}

In a two-species experiment, mw techniques are a very useful method to perform radiative evaporation, in particular when combined with other cooling techniques. Mw evaporation is very flexible with respect to the choice of the magnetic trap confining the atoms, because even for a large change of the local magnetic field determining the mw resonance, the shift of the mw reso- 
nance frequency remains within the emission bandwidth of the mw source. We showed that both $\mathrm{Rb}$ and $\mathrm{Cs}$ atoms can be efficiently evaporated using mw radiation. For a Rb-Cs mixture the insertion of a Cs mw evaporation stage allowed us to reach temperatures in the $15 \mu \mathrm{K}$ range for both species. A similar temperature could be reached through sympathetic cooling only wasting approximatively 50 percent of the $\mathrm{Rb}$ atoms. The combination of circle-ofdeath, mw and rf evaporative cooling allowed us to perform collisional studies of the Rb-Cs mixture and to derive the value of the interspecies scattering length, around $590 \mathrm{a}_{0}[12]$. As the main difference between rf and mw evaporation, owing to the presence of multiphoton transitions the rf field depletes all Zeeman sublevels of a target hyperfine state. By contrast, the mw evaporation is Zeeman sublevel selective. In fact we have made use of this selectivity associated with the mw radiation to remove either $\mathrm{Rb}$ or Cs atoms from specific Zeeman sublevels, manipulating and purifying the magnetization of our Rb-Cs mixture. Making use of the differential gravitational sag or of the SternGerlach separation we verified the efficiency of the mw selective evaporation. On the basis of precise simulations for the mw or rf evaporation, a quantitative comparison between the efficiencies reached in $\mathrm{rf}$ and mw evaporation could be performed. Furthermore additional information on the elastic, and inelastic, collisional properties of the ultracold mixture could be derived. However owing to the complexity of atomic Zeeman/hyperfine level structure such a simulation represents a very difficult task. Finally, we used mw spectroscopy to precisely calibrate the ellipticity of the rotating bias field of a TOP trap. All these applications of the mw evaporation are useful for the preparation of an atomic mixture in precisely controlled conditions.

\section{Acknowledgements}

We thank J. Reichel for preliminary discussions on the microwave equipment, A. Piombini for the network analyzer measurements, M. Giordano for the loan of microwave equipment, and A. Alberti and G. Tumino for help in the early stages of the experiment. This research was supported by the INFM (PRA Photonmatter), and by the EU Network Cold Quantum Gases.

\section{References}

[1] For a review of early experiments on Bose-Einstein condensation, see W. Ketterle, D.S. Durfee and D.M. Stamper-Kurn, in "Bose-Einstein condensation in atomic gases, Proceedings of the International School of Physics 'Enrico Fermi', Course CXL", (ed. M. Inguscio, S. Stringari and C.E. Wieman), IOS Press (Amsterdam), 67 (1999). 
[2] A. Mosk, S. Kraft, M. Mudrich, K. Singer, W. Wohlleben, R. Grimm, and M. Weidemüller, Appl. Phys. B 73, 791 (2001).

[3] G. Modugno, G. Ferrari, G. Roati, R.J. Brecha, A. Simoni, M. Inguscio, Science 294, 1320 (2001).

[4] G. Ferrari, M. Inguscio, W. Jastrzbeski, G. Modugno, G. Roati, and A. Simoni, Phys. Rev. Lett. 89, 053202 (2002).

[5] J. Goldwin, S. Inouye, M.L. Olsen, B. Newman, B.D. DePaola, and D.S. Jin, Phys. Rev. A 70, 021601(R) (2004).

[6] Z. Hadzibabic, C.A. Stan, K. Dieckmann, S. Gupta, M.W. Zwierlein, A. Görlitz, and W. Ketterle, Phys. Rev. Lett. 88, 160401 (2002).

[7] C.A. Stan, M.W. Zwierlein, C.H. Schunck, S.M.F. Raupach, and W. Ketterle, Phys. Rev. Lett. 93, 143001 (2004).

[8] C. Chin, V. Vuletić, A.J. Kerman, and S. Chu, Phys. Rev. Lett. 85, 2717 (2000). Communicated by C. Chin (private communication).

[9] A.G. Truscott, K.E. Strecker, W.I. McAlexander, G.B. Partridge and R.G. Hulet, Science 2912570 (2001).

[10] Z. Hadzibabic, C. A. Stan, K. Dieckmann, S. Gupta, M. W. Zwierlein, A. Grlitz, and W. Ketterle, Phys. Rev. Lett. 88, 160401 (2002).

[11] F. Schreck, L. Khaykovich, K. L. Corwin, G. Ferrari, T. Bourdel, J. Cubizolles, and C. Salomon, Phys. Rev. Lett. 87, 080403 (2001).

[12] M. Anderlini, E. Courtade, M. Cristiani, D. Cossart, D. Ciampini, C. Sias, O. Morsch, and E. Arimondo, Phys. Rev. A 71, 061401(R) (2005).

[13] In [14] the relative occupation of the ${ }^{40} \mathrm{~K}$ Zeeman levels was controlled by mw evaporation.

[14] B. DeMarco, J. L. Bohn, J. P. Burke, Jr., M. Holland, and D. S. Jin, Phys. Rev. Lett. 82, 4208 (1999)

[15] J.H. Müller, O. Morsch, D. Ciampini, M. Anderlini, R. Mannella and E. Arimondo, J. Phys. B: Atom. Mol. Opt. Phys. 33, 4095 (2000).

[16] On surface-wave antennas see Antenna Handbook, edited by Y.T. Loo and S.W. Lee, Van Nostrand Reinhold Company New York (1988).

[17] R. B. Watson and C. W. Horton, J. App. Phys. 19, 661 (1948); R. B. Watson and C. W. Horton, ibid. 19, 836 (1948).

[18] M.R. Matthews Ph.D. thesis, JILA University of Colorado at Boulder (CO).

[19] D.M. Harber, H.J. Lewandowski, J.M. McGuirk, and E.A. Cornell, Phys. Rev. A 66, 053616 (2002).

[20] P. Treutlein, P. Hommelhoff, T. Steinmetz, T.W. Hänsch, and J. Reichel, Phys. Rev. Lett, 92, 203005 (2004). 
[21] J.L. Martin, C.R. McKenzie, N.R. Thomas, D.M. Warrington and A.C. Wilson, J. Phys. B: At. Mol. Opt. Phys. 33, 3919 (2000).

[22] J.H. Müller, O. Morsch, D. Ciampini, M. Anderlini, R. Mannella and E. Arimondo, Phys. Rev. Lett. 85, 4454 (2000).

[23] J. Arlt, P. Bance, S. Hopkins, J. Martin, S. Webster, A. Wilson, K. Zetie and C.J. Foot, J. Phys. B: At. Mol. Opt. Phys. 31, L321 (1998).

[24] M. Arndt, M. Ben Dahan, D. Guéry-Odelin, M.W. Reynolds, and J. Dalibard, Phys. Rev. Lett. 79, 625 (1997). 